\section{POPULATION TESTING AND PERSONALISED OVARIAN CANCER RISK PREDICTION FOR RISK ADAPTED TARGETED PREVENTION}

${ }^{1}$ Faiza Gaba, ${ }^{2}$ Oleg Blyuss, ${ }^{3}$ Saskia Sanderson, ${ }^{4}$ Antonis Antoniou, ${ }^{5}$ Jatinderpal Kalsi, ${ }^{6}$ Andrew Wallace, ${ }^{7}$ Usha Menon, ${ }^{8}$ Rosa Legood, ${ }^{9}$ lan Jacobs, ${ }^{10}$ Ranjit Manchanda. 'Wolfson Institute of Preventative Medicine, Barts Cruk Cancer Centre, Queen Mary University of London; Centre for Cancer Prevention; ${ }^{2}$ University of Hertfordshire; School of Physics, Astronomy and Mathematics; ${ }^{3}$ University College London; Department of Behavioural Science and Health; "University of Cambridge; Centre for Cancer Genetic Epidemiology, Department of Public Health and Primary Care; ${ }^{5}$ University College London; Department of Women's Cancer, Elizabeth Garrett Anderson Institute for Women's Health; ${ }^{6}$ University of Manchester; Manchester Centre for Genomic Medicine; ' University College London; Medical Research Council Clinical Trials Unit at Ucl, Institute of Clinical Trials and Methodology; ${ }^{8}$ London School of Hygiene and Tropical Medicine; Department of Health Services Research and Policy; ${ }^{9}$ University of New South Wales; Department of Women's Health; ${ }^{10}$ Wolfson Institute of Preventative Medicine, Barts Cruk Cancer Centre, Queen Mary University of London; Department of Gynaecological Oncology, Barts Health NHS Trust; Centre for Cancer Prevention

10.1136/ijgc-2020-ESG0.159

Faiza Gaba, Oleg Blyuss, Jatinderpal Kalsi, Saskia Sanderson, Andrew Wallace, Antonis C. Antoniou, Rosa Legood, Usha Menon, Ian Jacobs, \& Ranjit Manchanda, on behalf of the PROMISE-FS team

Introduction/Background The current approach to genetic-testing and risk assessment is based on family-history and misses the majority of people at risk. Unselected population-based testing can enable personalised ovarian cancer (OC) risk prediction combining genetic/epidemiology/hormonal data. This permits population risk stratification for risk adapted targeted screening and prevention. Such an intervention study has not previously been undertaken. We aimed to assess the feasibility of OC risk stratification of general population women using a personalised OC risk tool followed by risk management.

Methodology Volunteers were recruited through London primary care networks. Inclusion criteria: women $\geq 18$ years. Exclusion criteria: prior ovarian/tubal/peritoneal cancer, previous genetic testing for OC genes. Participants accessed an online/web-based decision aid along with optional telephone helpline use. Consenting individuals completed risk assessment and underwent genetic testing (BRCA1/BRCA2/ RAD51C/RAD51D/BRIP1, OC susceptibility single-nucleotide polymorphisms). A validated OC risk prediction algorithm provided a personalised OC risk estimate using genetic/lifestyle/hormonal OC risk factors. Population genetic testing (PGT) for OC-risk stratification uptake/acceptability, satisfaction, decision aid/telephone helpline use, psychological health and quality of life were assessed using validated/customised questionnaires over six months. Linear-mixed models/contrast tests analysed impact on study outcomes. Main outcomes: feasibility/acceptability, uptake, decision aid/telephone helpline use, satisfaction/regret, and impact on psychological health/ quality of life.

Results In total, 123 volunteers (mean age $=48.5(\mathrm{SD}=15.4)$ years) used the decision aid, 105 (85\%) consented. None fulfilled NHS genetic-testing clinical criteria. OC-risk stratification revealed $1 / 103$ at $\geq 10 \%$ (high), $0 / 103$ at $\geq 5 \%-<10 \%$ (intermediate), and 100/103 at $<5 \%$ (low) lifetime OC risk. Decision aid satisfaction was $92.2 \%$. The telephone helpline use rate was $13 \%$ and the questionnaire response rate at six months was $75 \%$. The high-risk woman underwent surgical prevention. Contrast tests indicated that overall depression $(p=0.30)$, anxiety $(p=0.10)$, quality-of-life $(p=0.99)$, and distress $(p=0.25)$ levels did not jointly change, while OC worry $(\mathrm{p}=0.021)$ and general cancer risk perception $(p=0.015)$ decreased over six months. In total, $85.5 \%-98.7 \%$ were satisfied with their decision.

Conclusion Findings suggest population-based personalised OC risk stratification is feasible and acceptable, has high satisfaction, reduces cancer worry/risk perception, and does not negatively impact psychological health or quality-of-life. Larger implementation studies evaluating long-term impact and cost effectiveness of this strategy are needed.

Disclosures RM- funding from CRUK \& Eve Appeal for thiswork. Funding from Barts Charity, Rosetrees trust outside this work. Honorarium from Astrazeneca \& MSD.

IJ, UM- Financial interest in Abcodia, company for development of biomarkers for early detection of cancer. Other authors- No disclosures.

\section{ATTITUDES TOWARDS RISK REDUCING EARLY SALPINGECTOMY WITH DELAYED OOPHORECTOMY FOR OVARIAN CANCER PREVENTION: A COHORT STUDY}

${ }^{1}$ Faiza Gaba, ${ }^{2}$ Oleg Blyuss, ${ }^{3}$ Rosa Legood, ${ }^{4}$ Louise Izatt, ${ }^{4}$ Vishakha Tripathi, ${ }^{5}$ Kalpana Ragupathy, ${ }^{6}$ Robin Crawfordd, ${ }^{7} \mathrm{D}$ Gareth Evans, ${ }^{8}$ Usha Menon, ${ }^{1}$ Ranjit Manchanda. 'Wolfson Institute of Preventive Medicine; Barts Health NHS Trust; Gynaecological Oncology; ${ }^{2}$ Wolfson Institute of Preventive Medicine; ${ }^{3}$ London School of Hygiene and Tropical Medicine; ${ }^{4}$ Guy's Hospital; ${ }^{5}$ NHS Tayside; ${ }^{6}$ Cambridge University Hospitals NHS Foundation Trust; ${ }^{7}$ University of Manchester; ${ }^{8}$ University College London

\subsection{6/ijgc-2020-ESG0.160}

Introduction/Background With increasing evidence and acceptability of the central role of the fallopian-tube in the etiopathogeneisis of epithelial ovarian cancer (OC), risk-reducingearly-salpingectomy-and-delayed-oophorectomy (RRESDO) has been proposed as a two-stage surgical alternative to risk reducing salpingo-oophorectomy (RRSO). RRESDO offers some level of risk reduction to women who decline/wish to delay RRSO whilst conserving ovarian function and avoiding detrimental consequences of premature-menopause. However, prospective outcome data for RRESDO are lacking. The aim of this study was to determine RRESDO acceptability and effect of surgical prevention on menopausal sequelae/satisfaction/regret in women at increased OC risk.

Methodology UK Multicentre, cohort, study (IRSCTN:12310993). OC unaffected UK women $\geq 18$ years, at increased OC-risk, with/without previous RRSO, ascertained through specialist familial-cancer/genetic-clinics and BRCA support-groups. High-risk women completed a 39-item customised questionnaire developed through literature review, expert clinician and patient support groups' involvement. Baseline characteristics were described using descriptive statistics. Logistic/ linear-regression models analysed impact of variables on RRESDO acceptability and health-outcomes. Main outcomes were RRESDO acceptability, barriers/facilitators, menopausalsequelae, satisfaction/regret.

Results 346 of 683 participants underwent risk-reducing salpingo-oophorectomy (RRSO) and 337 did not. 69.1\% (181/ 262) premenopausal women who had not undergone RRSO found it acceptable to participate in a research study offering RRESDO. Premenopausal women concerned about sexual-dysfunction were more likely $(\mathrm{OR}=2.9, \quad 95 \% \mathrm{CI}=1.2-7.7$, $\mathrm{p}=0.025)$ to find RRESDO acceptable. Women experiencing sexual-dysfunction after premenopausal-RRSO were more 